Institute of $\mathbf{F}_{\text {ood and }} \mathbf{A g r i c u l t u r a l}_{\text {ciences }}$

\title{
Healthy Meal Plans ${ }^{1}$
}

Linda B. Bobroff ${ }^{2}$

\section{What Is a Meal Plan?}

A meal plan is a guide to help you plan daily meals and snacks. It allows you to eat foods that you enjoy and that provide a good balance of nutrients for your health. Meal plans can be used by anyone interested in healthy eating. They are very helpful for people who want to manage their weight.

\section{How Do Meal Plans Work?}

Each meal plan gives an example of one day's food intake using the food exchange system. The meal plans provide the recommended number of servings from the five food groups in the Food Guide Pyramid. Using one of the meal plans as a guide, you can select foods from the food exchange lists to plan healthful and tasty meals and snacks.

\section{How Does the Food Exchange System Work?}

In the food exchange system, foods are grouped into food lists according to their carbohydrate, protein, and fat content. Foods within each list contain similar amounts of total calories, carbohydrate, protein, and fat. The food exchange lists are:

$\begin{array}{ll}\text { - Starch } & \text { - Fat } \\ \text { - Fruit } & \text { - Free Foods } \\ \text { - Milk } & \text { - Combination } \\ & \text { Foods } \\ \text { - Other Carbohydrates } & \text { - Fast Foods } \\ \text { - Vegetable } & \\ \text { - Meat and Meat Substitutes }\end{array}$

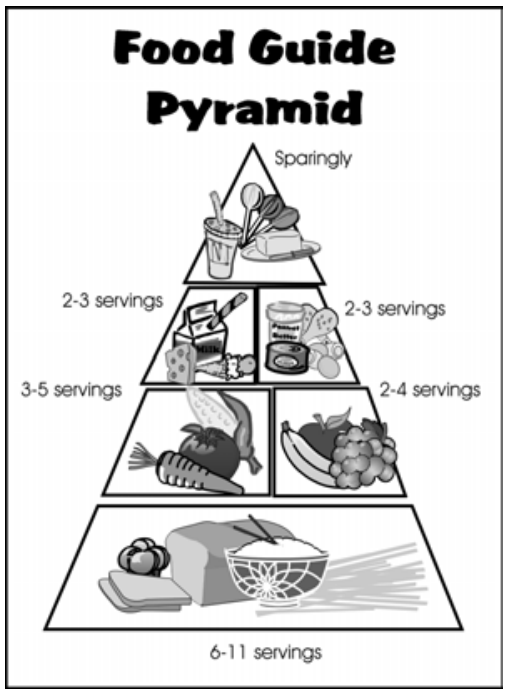

1. This document is FCS 8750, one of a series of the Department of Family, Youth and Community Sciences, Florida Cooperative Extension Service, Institute of Food and Agricultural Sciences, University of Florida. Originally published as HE8087. Publication date: June 2002. Please visit the EDIS Web site at http://edis.ifas.ufl.edu

2. Linda B. Bobroff, $\mathrm{PhD}, \mathrm{RD}, \mathrm{LD} / \mathrm{N}$, associate professor, Department of Family, Youth, and Community Sciences, Cooperative Extension Service, Institute of Food and Agricultural Sciences, University of Florida, Gainesville, 32611. Reviewed by R. Elaine Turner, PhD, RD, Jennifer Hillan, MSH, RD, LD/N, University of Florida, and Nancy J. Gal, MS, Marion County Extension Service educational information and other services only to individuals and institutions that function without regard to race, color, sex, age, handicap, or national origin. For information on obtaining other extension publications, contact your county Cooperative Extension Service office. Florida Cooperative Extension Service / Institute of Food and Agricultural Sciences / University of Florida / Christine Taylor Waddill, Dean 


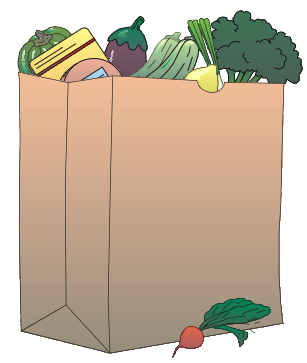

We recommend that you select a variety of foods that are "nutrient-dense." These foods provide plenty of vitamins and minerals along with calories (energy). Nutrient-dense foods are low in fat and added sugars. You can check food labels to see the fat and sugar content of foods. Low nutrient-density foods provide mostly calories with few other nutrients. We suggest that you eat these foods less often.

Limit higher fat foods to stay at your target calorie level. Ounce for ounce, fat provides more than twice as many calories as carbohydrate or protein. Foods that are high in fat also tend to be high in calories. But check the food label since some lowfat foods are high in calories too!

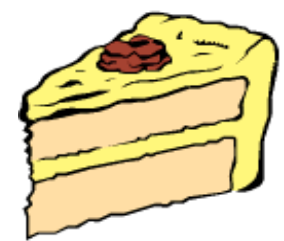

Hint: Be sure to choose foods that you enjoy! It will help you stick with your eating plan.

What about high calorie foods that you like to eat? Don't worry! You can eat these foods and still stay within your calorie goal. Think of foods like candy, cakes, cookies, and salted chips as "sometimes" foods. Have them once in a while and eat small portions.

\section{Setting up Your Meal Plan}

First, decide your daily calorie goal. This may be to maintain your weight or to lose weight. If you want to lose weight, select your calorie goal to lose no more than one-half to one pound per week. For most people, slow weight loss is best. For help in deciding your calorie goal, contact your county Extension family and consumer sciences agent, a registered dietitian (RD), or a public health nutritionist.

$$
\begin{aligned}
& \text { My Calorie goal to } \\
& \text { maintain my weight: } \\
& 1,500 \text { Calories per day }
\end{aligned}
$$

Next, look at the meal plan closest to your calorie level and then plan menus for one week. You can use the sample menu as a guide. Choose foods that are available and that you enjoy eating. Plan meals that will work for your lifestyle.

Include healthful snacks in your meal plan. They will help keep you from getting too hungry during the day. And be sure to drink at least six glasses of water every day. This will help to keep you "regular" and prevent problems as you eat more high-fiber foods.

As you begin selecting foods based on these meal plans, think of it as beginning a new way of life. Be sure to enjoy your meals, and invite your family and friends to share your new way of eating.

Finally, include physical activity in your healthier lifestyle. Being active will help you maintain a healthy body weight and keep you feeling healthy and strong. It also reduces health risks. If you have not been active for a long time, check with your doctor before you start an exercise program.

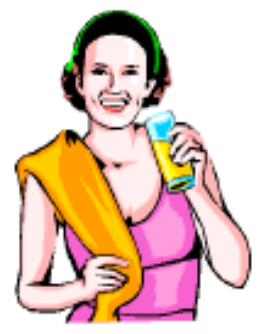




\section{Using Food Exchange Lists}

Complete food exchange lists are available in the publication Exchange Lists for Weight

Management (see last page for ordering information). The following lists will get you started.

\section{Starch List}

\section{1 exchange provides:}

15 grams carbohydrate

3 grams protein

0-1 gram fat

80 calories

Starch exchanges include bread, cereals, starchy vegetables, crackers and snacks, and dried beans, peas, and lentils. Most of these foods are found at the bottom of the Food Guide Pyramid. That means that we need the most servings of food from this group. These foods provide a variety of vitamins and minerals. One starch exchange is:

- $1 / 2$ cup of cereal, pasta, or starchy vegetable

- 1 ounce of bread

- $3 / 4$ to 1 ounce of most snack foods

\section{A few examples of one starch exchange are:}

$\begin{array}{ll}\text { Bread } & 1 \text { slice } \\ \text { Bagel } & 1 / 2 \\ \text { Cereals } & 1 / 2 \text { cup } \\ \text { Pasta } & 1 / 2 \text { cup, cooked } \\ \text { Baked beans } & 1 / 3 \text { cup } \\ \text { Rice } & 1 / 3 \text { cup, cooked } \\ \text { Corn } & 1 / 2 \text { cup } \\ \text { Potato, baked or boiled } & 1 \text { small } \\ \text { Popcorn (no fat added) } & 3 \text { cups } \\ \text { Snack chips, fat-free } & 15-20(3 / 4 \text { oz }) \\ \text { Beans and peas (dried) } & 1 / 2 \text { cup, cooked }\end{array}$

Some starchy foods are prepared with fat so they count as 1 starch and 1 fat exchange:

Corn bread, 2-in. cube 1 piece

French fried potatoes $\quad 16-25(3 \mathrm{oz})$

Taco shell, 6 in. $\quad 2$

Waffle, $4 \frac{1}{2}$ in. square 1

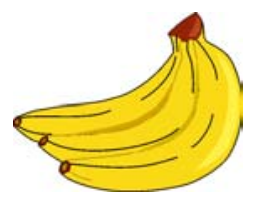

1 exchange provides:

15 grams carbohydrate

60 calories

Fruit exchanges include fresh, frozen, and canned fruits. When choosing canned fruit, select fruit canned in juice or extra light syrup. Include at least one fruit high in vitamin $\mathrm{C}$ (oranges, grapefruits, berries, and melons) every day. One fruit exchange is:

- 1 small to medium fresh fruit

- $1 / 2$ cup of canned or fresh fruit

- $1 / 2$ cup of fruit juice

\section{A few specific examples are:}

\begin{tabular}{ll} 
Banana & 1 small \\
Blueberries & $3 / 4$ cup \\
Cantaloupe & 1 cup cubes \\
Grapes, small & 17 \\
Pear, large & \multicolumn{1}{c}{$1 / 2$} \\
Plums, small & 2 \\
Prunes, dried & 3 \\
Strawberries & $11 \frac{1}{4}$ cup \\
Orange juice & $1 / 2$ cup
\end{tabular}

\section{Milk List}

1 exchange provides:

12 grams carbohydrate

8 grams protein

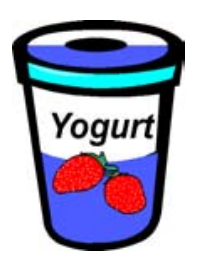


Milk and yogurt are excellent sources of calcium and protein as well as several vitamins. Select fat free or lowfat choices. One milk exchange is:

- 1 cup of fat free or $1 \%$ milk (see chart)

- $3 / 4$ cup of plain fat free yogurt

\begin{tabular}{||c|c|c||}
\hline $\begin{array}{c}\text { Type of milk } \\
\text { or yogurt }\end{array}$ & $\begin{array}{c}\text { Fat } \\
\text { (grams) }\end{array}$ & Calories \\
\hline \hline Fat Free & 0 & 80 \\
\hline Lowfat (1\%) & 2 & 100 \\
\hline Reduced Fat $(2 \%)$ & 5 & 120 \\
\hline Whole & 8 & 150 \\
\hline
\end{tabular}

Higher fat milk or yogurt $(2 \%$ and whole) counts as a milk exchange and 1 to $1 \frac{1 / 2}{2}$ fat exchanges.

\section{Other Carbohydrates List}

\section{1 exchange provides:}

15 grams carbohydrate or 1 starch, or 1 fruit, or 1 milk

Foods on this list include foods that contain added fat or sugar. You may substitute foods on this list for a starch, fruit, or milk serving. Keep in mind that these foods generally are less nutrient-dense than foods on the other lists. A few examples follow.

\section{Count as 1 carbohydrate:}

$\begin{array}{ll}\text { Cookie, fat-free } & 2 \text { small } \\ \text { Fruit juice bar, frozen } & 1 \mathrm{bar} \\ \text { Salad dressing, fat-free } & 1 / 4 \text { cup }\end{array}$

\section{Count as 1 carbohydrate and 1 fat:}

Brownie, small, unfrosted 2 in. square $\begin{array}{ll}\text { Granola bar } & 1 \text { bar } \\ \text { Ice cream, light } & 1 / 2 \text { cup }\end{array}$

\section{Vegetable List}

1 exchange provides:

5 grams carbohydrate

2 grams protein

25 calories

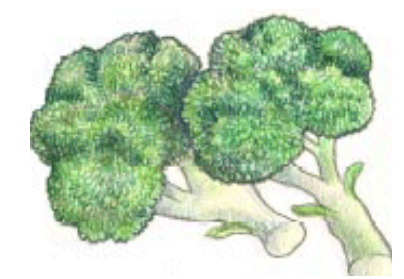

Vegetables include fresh, frozen, or canned vegetables. Canned vegetables often contain added salt; you can rinse and drain them to remove some salt. Deep green and orange vegetables provide vitamin A and other nutrients. Several vegetables, including peppers, broccoli, and tomatoes, are rich sources of vitamin $\mathrm{C}$.

Starchy vegetables like corn, peas, and potatoes are not included here; they are on the starch list. One vegetable exchange is:

- $1 / 2$ cup of cooked vegetables

- $1 / 2$ cup of vegetable juice

- 1 cup of raw vegetables

\section{Meat and Meat Alternates List}

\section{1 exchange provides:}

7 grams protein

$0-8$ grams fat

35-100 calories

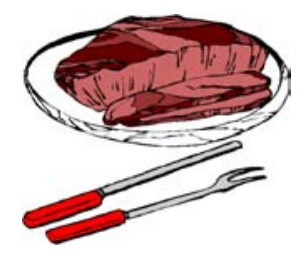

Meat and meat alternates are divided into four lists, depending on their fat content. Select very lean or lean meats and alternates most of the time. Use low-fat cooking methods like broiling, baking, and grilling to limit added fat. One meat exchange is:

- 1 oz meat, fish, poultry, or cheese

- $\quad 1 / 2$ cup dried beans (cooked)

- 1 egg

A typical portion of meat often includes three or more exchanges since an exchange is only a oneounce serving. 
Very lean meat and substitutes include white meat chicken or turkey, lean fish, fat-free cheese, egg whites and egg substitutes. Dried beans, peas, and lentils count as one very lean meat and one starch exchange.

Lean meat and substitutes include lean cuts of beef, pork, and other meats, dark meat chicken or turkey, high-fat fish, and cheeses with 1 to 3 grams of fat per ounce.

Medium-fat meat and substitutes include ground beef, ribs, poultry with skin, fried fish, eggs, and tofu.

High-fat meat and substitutes are high in fat, saturated fat, and cholesterol. This includes regular cheeses like cheddar, Swiss, and American. Also included are spare ribs, processed sandwich meats, and peanut butter.

\section{Fat List}

\section{1 exchange provides:}

5 grams fat

45 calories

The Dietary Guidelines recommend that less than 30 percent of our calories come from fat. The meal plans included in this publication provide about 20 to 25 percent of calories from fat.

All fats are concentrated in calories whether they are in solid, whipped, or liquid form. For this reason they need to be measured carefully. One tablespoon of margarine, butter, or mayonnaise contains 100 calories.

Also, it's important to limit saturated fat. Solid fats, like butter and lard, are high in saturated fat Instead, use oils like olive, canola, and peanut oils.
One fat exchange is:

- 1 teaspoon of margarine, butter, or oil

- 1 tablespoon of regular salad dressing

\section{A few examples of one fat exchange are:}

Avocado, medium $1 / 8$

Almonds or cashews

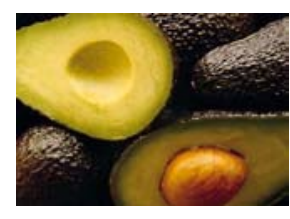

Peanut butter

6 nuts

Cream cheese

2 tsp

Bacon, cooked

1 Tbsp

1 slice

\section{Free Foods List}

Foods on this list contain less than 20 calories or less than 5 grams of carbohydrate per serving. When no serving size is given, you can eat the food as often as you desire. However, eat bouillon, pickles, and other high-sodium foods in moderation.

\section{Free foods are divided into 5 groups:}

- Fat free and reduced fat foods:

$\begin{array}{ll}\text { Cream cheese, fat free } & 1 \mathrm{Tbsp} \\ \text { Salad dressing, fat free } & 1 \mathrm{Tbsp} \\ \text { Margarine, reduced-fat } & 1 \mathrm{tsp}\end{array}$

- Sugar free or low sugar foods:

Candy, sugar free $\quad 1 \mathrm{pc}$

Gum, sugar free

Jam or jelly, low sugar 2 tsp

- Drinks: Bouillon, Coffee, Tea 
- Condiments:

$\begin{array}{ll}\text { Catsup } & 1 \text { Tbsp } \\ \text { Lemon juice } & \end{array}$

- Seasonings:

Herbs

Spices

\section{The Meal Plans}

We developed two sets of healthy meal plans to help you plan your daily food intake. The

Traditional Meal Plans include foods from all food groups. The Vegetarian Meal Plans omit meat, poultry, and fish. These meal plans include meat substitutes and dairy products, so they are appropriate for lacto-ovo vegetarians (those who eat milk products and eggs).

The meal plans range from approximately 1,200 to 2,500 calories. Dietitians recommend that you eat at least 1,200 calories each day and men at least 1,600 calories per day, even when trying to lose weight.

The number of calories you actually eat will depend upon several things:

- the exact foods you choose (for example, the cut of meat or type of bread)

- how you prepare the foods

- the exact amount of each food that you eat
Within each set of meal plans, higher calorie menus build on the foods in the 1,200 calorie meal plan. This way you can see which foods were added to increase the calorie level. When added foods first appear, they are marked with an asterisk (*). When the serving size of a food increases, it is marked with an asterisk too.

Work with your county Extension family and consumer sciences educator, a registered dietitian, or public health nutritionist to find the meal plan that will work for you.

\section{For More Information}

American Diabetes Association, Inc. and The American Dietetic Association. Exchange Lists for Weight Management. Revised - 1995. [To order, call the American Diabetes Association at (800) 232-3472.]

Contact your local county Extension office for information and resources. The Family and Consumer Sciences agent may offer classes in nutrition and other topics of interest. The Extension office is listed in the blue pages of your telephone book under county government.

Check out the following reliable websites:

Http://www.nal.usda.gov/fnic Http://www.nutrition.gov Http://fycs.ifas.ufl.edu Http://www.diabetes.org 


\section{Traditional Meal Plans}

1200 Calorie Meal Plan

7 Starch Exchanges

2 Fruit Exchanges

2 Milk Exchanges

Meal

Morning

Noon

$\begin{array}{ll} & 1 \text { Fat } \\ & 1 \text { Vegetable } \\ & \text { Free Food } \\ & \text { Free Food } \\ \text { Afternoon } & 1 \text { Milk } \\ & 1 \text { Fruit } \\ & \text { Free Food } \\ & 3 \text { Meat } \\ & 2 \text { Starch } \\ & 1 \text { Vegetable } \\ & 1 \text { Vegetable } \\ & \text { Free Food } \\ & 1 \text { Fat }\end{array}$

Late Night 1 Starch

1 Fat

$1 / 2$ Milk
3 Vegetable Exchanges

3 Meat Exchanges

4 Fat Exchanges

\section{Sample Menu for One Day}

1 small whole wheat bagel (or $1 / 2$ large)

1 Tbsp regular cream cheese

$1 / 2$ cup fat free milk

1 small orange

coffee or tea

2 slices whole wheat bread

1 teaspoon tub margarine

1 cup mixed salad greens

2 Tbsp fat free Italian salad dressing

iced tea, unsweetened

1 cup fat free plain yogurt

$1 / 2$ large banana

sugar substitute and vanilla extract, if desired

3 ounces broiled fish

$2 / 3$ cup cooked white rice

$1 / 2$ cup cooked greens (no added fat)

$1 / 2$ cup cooked summer squash

2 Tbsp fat free French salad dressing

1 teaspoon tub margarine

1 slice rye bread

1 teaspoon tub margarine

$1 / 2$ cup fat-free milk 


\section{Calorie Meal Plan}

\author{
8 Starch Exchanges \\ 3 Fruit Exchanges \\ 2 Milk Exchanges
}

Meal

Morning

$\begin{array}{ll}\text { Midmorning } & \text { Free Food } \\ & 1 \text { Starch } \\ & 1 \text { Fruit } \\ & 2 \text { Starch } \\ & 1 \text { Fat } \\ & 1 \text { Vegetable } \\ & 1 \text { Vegetable } \\ & \text { Free Food } \\ & \text { Free Food }\end{array}$

$\begin{array}{ll}\text { Afternoon } & 1 \text { Milk } \\ & 1 \text { Fruit } \\ & \text { Free Food }\end{array}$

$\begin{array}{ll}\text { Evening } & 3 \text { Meat } \\ & 2 \text { Starch } \\ & 1 \text { Vegetable } \\ & 1 \text { Vegetable } \\ & \text { Free Food } \\ & 1 \text { Fat }\end{array}$

$\begin{array}{ll}\text { Late Night } & 1 \text { Starch } \\ & 1 \text { Fat } \\ & 1 / 2 \text { Milk }\end{array}$

*added food items or increased amounts from previous meal plan.

\author{
4 Vegetable Exchanges \\ 3 Meat Exchanges \\ 4 Fat Exchanges
}

Sample Menu for One Day

1 small whole wheat bagel (or $1 / 2$ large)

1 Tbsp regular cream cheese

$1 / 2$ cup fat-free milk

1 small orange

coffee or tea

5 whole wheat crackers*

$1 / 2$ cup apple juice*

2 slices whole wheat bread

1 teaspoon tub margarine

1 cup mixed salad greens

1 cup cut-up raw vegetables*

2 Tbsp fat-free Italian salad dressing

iced tea, unsweetened

1 cup fat-free plain yogurt

$1 / 2$ large banana

sugar substitute and vanilla extract, if desired

3 ounces broiled fish

$2 / 3$ cup cooked white rice

$1 / 2$ cup cooked greens (no added fat)

$1 / 2$ cup cooked summer squash

2 Tbsp fat-free French salad dressing

1 teaspoon tub margarine

1 slice rye bread

1 teaspoon tub margarine

$1 / 2$ cup fat-free milk 


\section{Traditional Meal Plans}

\section{Calorie Meal Plan}

\author{
9 Starch Exchanges \\ 4 Fruit Exchanges \\ 2 Milk Exchanges
}

\begin{tabular}{ll} 
Meal & Meal Plan \\
\hline Morning & 2 Starch \\
& 1 Fat \\
& $1 / 2$ Milk \\
& 1 Fruit \\
& Free Food \\
& 2 Starch \\
Midmorning & 1 Meat and 1 Fat \\
& 1 Fruit \\
& 2 Starch \\
& 1 Fat \\
& 1 Vegetable \\
& 1 Vegetable \\
& Free Food \\
& Free Food
\end{tabular}

Afternoon 1 Milk

2 Fruit

Free Food

Evening 3 Meat

2 Starch

1 Vegetable

2 Vegetable

Free Food

1 Fat

Late Night

1 Starch
1 Fat
$1 / 2$ Milk

\author{
5 Vegetable Exchanges \\ 4 Meat Exchanges \\ 5 Fat Exchanges
}

\section{Sample Menu for One Day}

1 small whole wheat bagel (or $1 / 2$ large)

1 Tbsp regular cream cheese

$1 / 2$ cup fat-free milk

1 small orange

coffee or tea

10 whole wheat crackers *

1 ounce cheddar cheese*

$1 / 2$ cup apple juice

2 slices whole wheat bread

1 teaspoon tub margarine

1 cup mixed salad greens

1 cup cut-up raw vegetables

2 Tbsp fat-free Italian salad dressing

iced tea, unsweetened

1 cup fat-free plain yogurt

1 large banana*

sugar substitute and vanilla extract, if desired

3 ounces broiled fish

$2 / 3$ cup cooked white rice

$1 / 2$ cup cooked greens (no added fat)

1 cup cooked summer squash*

2 Tbsp fat-free French salad dressing

1 teaspoon tub margarine

1 slice rye bread

1 teaspoon tub margarine

$1 / 2$ cup fat-free milk

*added food items or increased amounts from previous meal plan 


\section{Calorie Meal Plan}

10 Starch Exchanges

4 Fruit Exchanges

2 Milk Exchanges

Meal

Morning

Morning

\section{Midmorning}

Meal Plan

2 Starch

1 Starch

1 Fat

$1 / 2$ Milk

1 Fruit

Free Food

Noon

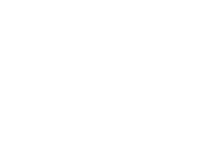

$\begin{array}{ll} & \text { Free Food } \\ & \text { Free Food } \\ \text { Afternoon } & 1 \text { Milk and 1 Fat } \\ & 2 \text { Fruit } \\ & \text { Free Food } \\ \text { Evening } & 4 \text { Meat } \\ & 2 \text { Starch } \\ & 2 \text { Vegetable } \\ & 2 \text { Vegetable } \\ & \text { Free Food } \\ & 1 \text { Fat } \\ & 1 \text { Starch } \\ & 1 \text { Fat } \\ & 1 / 2 \text { Milk }\end{array}$

\section{Traditional Meal Plans}

6 Vegetable Exchanges

5 Meat Exchanges

6 Fat Exchanges

\section{Sample Menu for One Day}

1 small whole wheat bagel (or $1 / 2$ large)

$1 / 2$ cup dry cereal ${ }^{*}$

1 Tbsp regular cream cheese

$1 / 2$ cup fat-free milk

1 small orange

coffee or tea

10 whole wheat crackers

1 ounce cheddar cheese

$1 / 2$ cup apple juice

2 slices whole wheat bread

1 teaspoon tub margarine

1 cup mixed salad greens

1 cup cut-up raw vegetables

2 Tbsp fat-free Italian salad dressing

iced tea, unsweetened

1 cup lowfat plain yogurt*

1 large banana

sugar substitute and vanilla extract, if desired

4 ounces broiled fish*

$2 / 3$ cup cooked white rice

1 cup cooked greens (no added fat)

1 cup cooked summer squash

2 Tbsp fat-free French salad dressing

1 teaspoon tub margarine

1 slice rye bread

1 teaspoon tub margarine

$1 / 2$ cup fat-free milk

* added food items or increased amounts from previous meal plan 


\section{Calorie Meal Plan}

11 Starch Exchanges

4 Fruit Exchanges

3 Milk Exchanges

\begin{tabular}{|c|c|c|}
\hline Meal & Meal Plan & Sample Menu for One Day \\
\hline \multirow[t]{6}{*}{ Morning } & 2 Starch & 1 small whole wheat bagel (or $1 / 2$ large) \\
\hline & 2 Starch & 1 cup dry cereal* \\
\hline & 1 Fat & 1 Tbsp regular cream cheese \\
\hline & 1 Milk & 1 cup fat-free milk* \\
\hline & 1 Fruit & 1 small orange \\
\hline & Free Food & coffee or tea \\
\hline \multirow[t]{3}{*}{ Midmorning } & 2 Starch & 10 whole wheat crackers \\
\hline & 1 Meat and 1 Fat & 1 ounce cheddar cheese \\
\hline & 1 Fruit & $1 / 2$ cup apple juice \\
\hline \multirow[t]{6}{*}{ Noon } & 2 Starch & 2 slices whole wheat bread \\
\hline & 1 Fat & 1 teaspoon tub margarine \\
\hline & 1 Vegetable & 1 cup mixed salad greens \\
\hline & 1 Vegetable & 1 cup cut-up raw vegetables \\
\hline & Free Food & 2 Tbsp fat-free Italian salad dressing \\
\hline & Free Food & iced tea, unsweetened \\
\hline \multirow[t]{3}{*}{ Afternoon } & 1 Milk and 1 Fat & 1 cup lowfat plain yogurt \\
\hline & 2 Fruit & 1 large banana \\
\hline & Free Food & sugar substitute and vanilla extract, if desired \\
\hline \multirow[t]{6}{*}{ Evening } & 4 Meat & 4 ounces broiled fish \\
\hline & 2 Starch & $2 / 3$ cup cooked white rice \\
\hline & 2 Vegetable & 1 cup cooked greens (no added fat) \\
\hline & 2 Vegetable & 1 cup cooked summer squash \\
\hline & 1 Fat & 2 Tbsp light ranch salad dressing* \\
\hline & 1 Fat & 1 teaspoon tub margarine \\
\hline \multirow[t]{3}{*}{ Late Night } & 1 Starch & 1 slice rye bread \\
\hline & 1 Fat & 1 teaspoon tub margarine \\
\hline & 1 Milk & 1 cup fat-free milk* \\
\hline
\end{tabular}

6 Vegetable Exchanges

5 Meat Exchanges

7 Fat Exchanges

\section{Traditional Meal Plans}

*added food items or increased amounts from previous meal plan 


\section{Calorie Meal Plan}

14 Starch Exchanges

5 Fruit Exchanges

3 Milk Exchanges

\section{Meal}

Morning

2 Starch

1 Fat

1 Milk

1 Fruit

Free Food

Midmorning 2 Starch

1 Meat and 1 Fat

2 Fruit

Noon

2 Starch

1 Fat

2 Vegetable

2 Starch

1 Fat

Free Food

Afternoon

1 Milk and 1 Fat

2 Fruit

1 Vegetable

Free Food

Evening

6 Meat

3 Starch

2 Vegetable

2 Vegetable

1 Fat

1 Fat

Late Night 1 Starch

1 Fat

1 Milk

\title{
Traditional Meal Plans
}

\author{
7 Vegetable Exchanges \\ 7 Meat Exchanges \\ 8 Fat Exchanges
}

\section{Sample Menu for One Day}

1 small whole wheat bagel (or $1 / 2$ large)

1 cup dry cereal

1 Tbsp regular cream cheese

1 cup fat-free milk

1 small orange

coffee or tea

10 whole wheat crackers

1 ounce cheddar cheese

1 cup apple juice*

2 slices wheat bread

1 teaspoon tub margarine

2 cups mixed salad greens

1 cup cooked pasta*

2 Tbsp lite ranch salad dressing*

iced tea, unsweetened

1 cup lowfat plain yogurt

1 large banana

1 carrot*

sugar substitute and vanilla extract, if desired

6 ounces broiled fish*

1 cup cooked white rice*

1 cup cooked greens (no added fat)

1 cup cooked summer squash

2 Tbsp light ranch salad dressing

1 teaspoon tub margarine

1 slice rye bread

1 teaspoon tub margarine

1 cup fat-free milk

*added food items or increased amounts from previous meal plan 


\section{Calorie Meal Plan}

\section{Vegetarian Meal Plans}

$\begin{array}{ll}6 \text { Starch Exchanges } & 4 \text { Vegetable Exchanges } \\ 3 \text { Fruit Exchanges } & 3 \text { Meat Exchanges } \\ 2 \text { Milk Exchanges } & 4 \text { Fat Exchanges }\end{array}$

\begin{tabular}{|c|c|c|}
\hline Meal & Meal Plan & Sample Menu for One Day \\
\hline \multirow[t]{5}{*}{ Morning } & 1 Starch & $1 / 2$ cup bran cereal \\
\hline & 1 Milk & 1 cup $1 \%$ low-fat milk \\
\hline & 1 Fruit & 1 medium orange \\
\hline & 1 Vegetable & $1 / 2$ cup tomato juice \\
\hline & Free Food & coffee or tea \\
\hline \multirow[t]{7}{*}{ Noon } & $\begin{array}{l}1 \text { Meat Substitute and } \\
2 \text { Fat }\end{array}$ & 2 tablespoons peanut butter \\
\hline & Free Food & 2 teaspoons low-sugar jam \\
\hline & 2 Starch & 2 slices wheat bread \\
\hline & 1 Fruit & $1 / 2$ cup apple juice \\
\hline & 1 Vegetable & 1 cup tossed green salad \\
\hline & Free Food & 2 Tbsp fat-free salad dressing \\
\hline & Free Food & iced tea, unsweetened \\
\hline \multirow[t]{3}{*}{ Afternoon } & 1 Milk and $1 / 2$ Fat & $3 / 4$ Cup low-fat plain yogurt \\
\hline & 1 Fruit & $11 / 4$ cup whole strawberries, sliced \\
\hline & Free Food & sugar substitute and vanilla extract, if desired \\
\hline \multirow[t]{5}{*}{ Evening } & $\begin{array}{l}2 \text { Meat Substitute and } \\
2 \text { Starch }\end{array}$ & 1 cup black beans \\
\hline & 1 Starch & $1 / 3$ cup cooked white rice \\
\hline & 1 Fat & 1 teaspoon olive oil \\
\hline & 1 Vegetable & $1 / 2$ cup cooked broccoli \\
\hline & $1 / 2$ Fat & $1 / 2$ teaspoon margarine \\
\hline Late Night & 1 Vegetable & 1 cup baby carrots, raw \\
\hline
\end{tabular}




\section{Calorie Meal Plan}

\begin{tabular}{|c|c|c|}
\hline Meal & Meal Plan & Sample Menu for One Day \\
\hline \multirow[t]{7}{*}{ Morning } & 1 Starch & $1 / 2$ cup bran cereal \\
\hline & 1 Milk & 1 cup $1 \%$ low-fat milk \\
\hline & 1 Fruit & 1 medium orange \\
\hline & 1 Starch & 1 slice rye toast ${ }^{*}$ \\
\hline & 1 Fat & 1 teaspoon margarine* \\
\hline & 1 Vegetable & $1 / 2$ cup tomato juice \\
\hline & Free Food & coffee or tea \\
\hline \multirow[t]{6}{*}{ Noon } & $\begin{array}{l}1 \text { Meat Substitute } \\
\text { and } 2 \text { Fat }\end{array}$ & 2 tablespoons peanut butter \\
\hline & Free Food & 2 teaspoons low-sugar jam \\
\hline & 2 Starch & 2 slices wheat bread \\
\hline & 2 Fruit & 1 cup apple juice* \\
\hline & 1 Vegetable & 1 cup tossed green salad \\
\hline & Free Food & 2 Tbsp fat-free salad dressing \\
\hline \multirow[t]{3}{*}{ Afternoon } & 1 Milk and $1 / 2$ Fat & $3 / 4$ cup low-fat plain yogurt \\
\hline & 1 Fruit & $11 / 4$ cup whole strawberries, sliced \\
\hline & Free Food & sugar substitute and vanilla extract, if desired \\
\hline \multirow[t]{7}{*}{ Evening } & $\begin{array}{l}2 \text { Meat Substitutes } \\
\text { and } 2 \text { Starch }\end{array}$ & 1 cup black beans \\
\hline & 1 Starch & $1 / 3$ cup cooked white rice \\
\hline & 1 Fat & 1 teaspoon olive oil \\
\hline & 1 Vegetable & $1 / 2$ cup cooked broccoli \\
\hline & $1 / 2$ Fat & $1 / 2$ teaspoon margarine \\
\hline & 1 Vegetable & 1 cup tossed green salad* \\
\hline & Free Food & 2 Tbsp fat-free salad dressing* \\
\hline Late Night & 1 Vegetable & 1 cup baby carrots, raw \\
\hline
\end{tabular}

7 Starch Exchanges

4 Fruit Exchanges

2 Milk Exchanges

\section{Vegetarian Meal Plans}

5 Vegetable Exchanges

3 Meat Exchanges

5 Fat Exchanges

\section{Sample Menu for One Day}

1 medium orange

Free Food

1 Meat Substitute

2 Fat

2 tablespoons peanut butter

teaspoons low-sugar jam

at bread

1 cup tossed green salad

2 Tbsp fat-free salad dressing

sugar substitute and vanilla extract, if desired

1 teaspoon olive oil

coup cood broccoli

sing

1 cup baby carrots, raw *added food items or increased amounts from previous meal plan 


\section{Calorie Meal Plan}

9 Starch Exchanges

4 Fruit Exchanges

2 Milk Exchanges

Meal

Morning

(a)

.

Noon

Noon

$\begin{array}{ll} & \text { and } 2 \text { Fat } \\ & \text { Free Food } \\ & 2 \text { Starch } \\ & 2 \text { Fruit } \\ & 1 \text { Vegetable } \\ & \text { Free Food } \\ \text { Afternoon } & 1 \text { Milk and } 1 / 2 \text { Fat } \\ & 1 \text { Fruit } \\ & \text { Free Food }\end{array}$

Evening

2 Meat Substitutes and 2 Starch

1 Starch

1 Fat

1 Vegetable

$1 / 2$ Fat

1 Vegetable

Free Food

Late Night 1 Vegetable

2 Starch

Free Food

\section{Vegetarian Meal Plans}

5 Vegetable Exchanges

4 Meat Exchanges

5 Fat Exchanges

Sample Menu for One Day

$1 / 2$ cup bran cereal

1 cup $1 \%$ low-fat milk

1 medium orange

2 egg whites or $1 / 4$ cup egg substitute, scrambled*

1 slice rye toast

1 teaspoon margarine

$1 / 2$ cup tomato juice

coffee or tea

2 tablespoons peanut butter

2 teaspoons low-sugar jam

2 slices wheat bread

1 cup apple juice

1 cup tossed green salad

2 Tbsp fat-free salad dressing

$3 / 4$ cup low-fat plain yogurt

$11 / 4$ cup whole strawberries, sliced

sugar substitute and vanilla extract, if desired

1 cup black beans

$1 / 3$ cup cooked white rice

1 teaspoon olive oil

$1 / 2$ cup cooked broccoli

$1 / 2$ teaspoon margarine

1 cup tossed green salad

2 Tbsp fat-free salad dressing

1 cup baby carrots, raw

1 small bagel ${ }^{*}$

2 teaspoons low-sugar jam*

*added food items or increased amounts from previous meal plan 
1800 Calorie Meal Plan

\begin{tabular}{|c|c|c|}
\hline \multirow[b]{4}{*}{ Meal } & 9 Starch Exchanges & 5 Vegetable Exchanges \\
\hline & 5 Fruit Exchanges & 4 Meat Exchanges \\
\hline & 3 Milk Exchanges & 6 Fat Exchanges \\
\hline & Meal Plan & Sample Menu for One Day \\
\hline \multirow[t]{8}{*}{ Morning } & 1 Starch & $1 / 2$ cup bran cereal \\
\hline & 1 Milk & 1 cup $1 \%$ low-fat milk \\
\hline & 1 Fruit & 1 medium orange \\
\hline & 1 Meat Substitute & 2 egg whites or $1 / 4$ cup egg substitute, scrambled \\
\hline & 1 Starch & 1 slice rye toast \\
\hline & 1 Fat & 1 teaspoon margarine \\
\hline & 1 Vegetable & $1 / 2$ cup tomato juice \\
\hline & Free Food & coffee or tea \\
\hline \multirow[t]{6}{*}{ Noon } & $\begin{array}{l}1 \text { Meat Substitute } \\
\text { and } 2 \text { Fat }\end{array}$ & 2 tablespoons peanut butter \\
\hline & Free Food & 2 teaspoons low sugar jam \\
\hline & 2 Starch & 2 slices wheat bread \\
\hline & 2 Fruit & 1 cup apple juice \\
\hline & 1 Vegetable & 1 cup tossed green salad \\
\hline & Free Food & 2 Tbsp fat-free salad dressing \\
\hline \multirow[t]{3}{*}{ Afternoon } & 1 Milk and $1 / 2$ Fat & $3 / 4$ cup low-fat plain yogurt \\
\hline & 1 Fruit & $1 \frac{1}{4}$ cup whole strawberries, sliced \\
\hline & Free Food & sugar substitute and vanilla extract, if desired \\
\hline \multirow[t]{8}{*}{ Evening } & $\begin{array}{l}2 \text { Meat Substitutes } \\
\text { and } 2 \text { Starch }\end{array}$ & 1 cup black beans \\
\hline & 1 Starch & $1 / 3$ cup cooked white rice \\
\hline & 1 Fat & 1 teaspoon olive oil \\
\hline & 1 Vegetable & $1 / 2$ cup cooked broccoli \\
\hline & $1 / 2$ Fat & $1 / 2$ teaspoon margarine \\
\hline & 1 Vegetable & 1 cup tossed green salad \\
\hline & Free Food & 2 Tbsp fat-free salad dressing \\
\hline & 1 Fruit & $1 / 2$ cup cranberry juice cocktail* \\
\hline \multirow[t]{4}{*}{ Late Night } & 1 Vegetable & 1 cup baby carrots, raw \\
\hline & 2 Starch & 1 small bagel \\
\hline & 1 Fat & 2 Tbsp reduced fat cream cheese* \\
\hline & 1 Milk & 1 cup $1 \%$ low-fat milk* \\
\hline
\end{tabular}

*added food items or increased amounts from previous meal plan 


\section{Calorie Meal Plan}

10 Starch Exchanges

5 Fruit Exchanges

3 Milk Exchanges

\begin{tabular}{ll} 
Meal & Meal Plan \\
\hline Morning & 2 Starch \\
& 1 Milk \\
& 1 Fruit \\
& 1 Meat Substitute \\
& 1 Starch \\
& 1 Fat \\
& 1 Vegetable \\
& Free Food \\
& 1 Meat Substitute \\
& and 2 Fat \\
& Free Food \\
& 2 Starch \\
& 2 Fruit \\
& 1 Vegetable \\
& Free Food \\
& 1 Milk and $1 / 2$ Fat \\
& 1 Fruit \\
& Free Food \\
& 1 Meat Substitute \\
Afternoon & and 1 Fat \\
& 2 Meat Substitute \\
& and 2 Starch \\
& 1 Starch \\
& 1 Fat \\
& 1 Vegetable \\
& $1 / 2$ Fat \\
& 1 Vegetable \\
& Free Food \\
& 1 Fruit \\
& 1 Evening
\end{tabular}

Late Night 1 Vegetable

2 Starch

1 Fat

1 Milk

\section{Vegetarian Meal Plans}

5 Vegetable Exchanges

5 Meat Exchanges

7 Fat Exchanges

\section{Sample Menu for One Day}

1 cup bran cereal ${ }^{*}$

1 cup $1 \%$ low-fat milk

1 medium orange

2 egg whites or $1 / 4$ cup egg substitute, scrambled

1 slice rye toast

1 teaspoon margarine

$1 / 2$ cup tomato juice

coffee or tea

2 tablespoons peanut butter

2 teaspoons low sugar jam

2 slices wheat bread

1 cup apple juice

1 cup tossed green salad

2 Tbsp fat-free salad dressing

$3 / 4$ cup low-fat plain yogurt

$1 \frac{1}{4}$ cup whole strawberries, sliced

sugar substitute and vanilla extract, if desired

1 ounce hard cheese (like cheddar) ${ }^{*}$

1 cup black beans

$1 / 3$ cup cooked white rice

1 teaspoon olive oil

$1 / 2$ cup cooked broccoli

$1 / 2$ teaspoon margarine

1 cup tossed green salad

2 Tbsp fat-free salad dressing

$1 / 2$ cup cranberry juice cocktail

1 cup baby carrots, raw

1 small bagel

2 Tbsp reduced fat cream cheese

1 cup $1 \%$ low-fat milk

*added food items or increased amounts from previous meal plan 